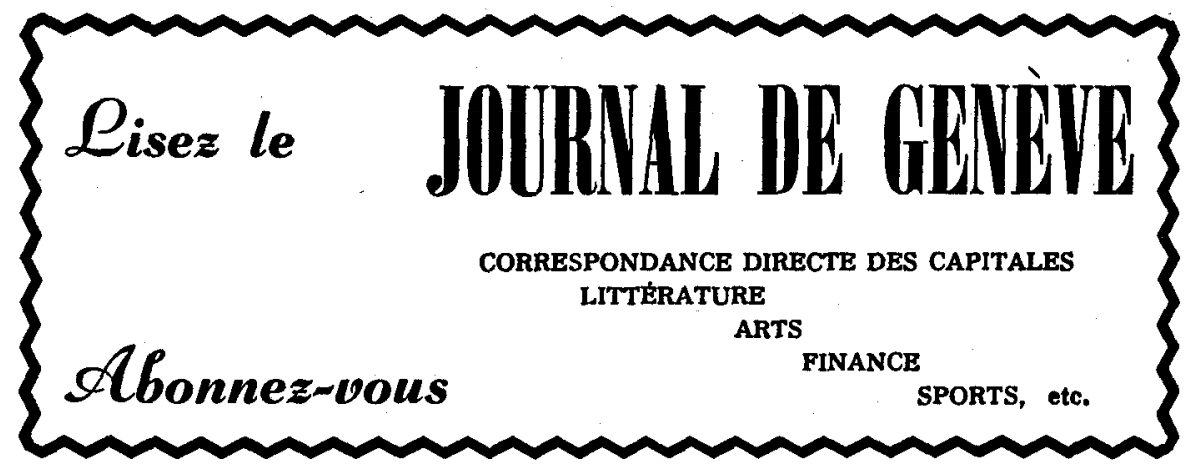

\title{
HEBERLÉ S.A.
}

FOURNITURES INDUSTRIELLES EN GROS

GENEVE

Rue du Rhone of et Grand-aual 42 T6lophone 41377 (2 lipnes)

Amiante

Bottes en caoutchouc

Chiffons d'essuyages blanes et couleurs

Courroles de transmissions caoutchouc, cuir ou tissees

Déchets de coton d'essuyage blancs et couleurs

Eponges

Eponges naturelles et viscose

Feutre industriel

Joints en tous genres

Peaux de daim

Produits abrasifs - SIA .

Tabliers industriels en caoutchouc, cuir, GURIT, toile

Tapis en caoutchouc

Tuyaux d'arrosage, à air comprimé, à vin, à vapeur, pour radiateurs

Tous tuyaux caoutchouc pour

l'industrie
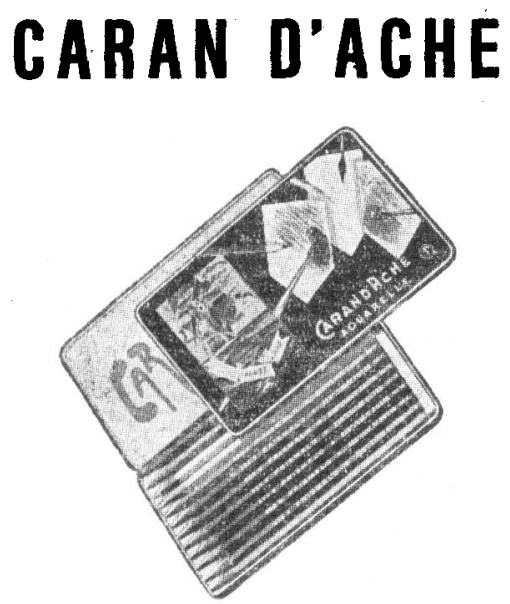

PRISMALO 999

Lo meillen crayson do couteun

$$
\text { pour l'écolo }
$$

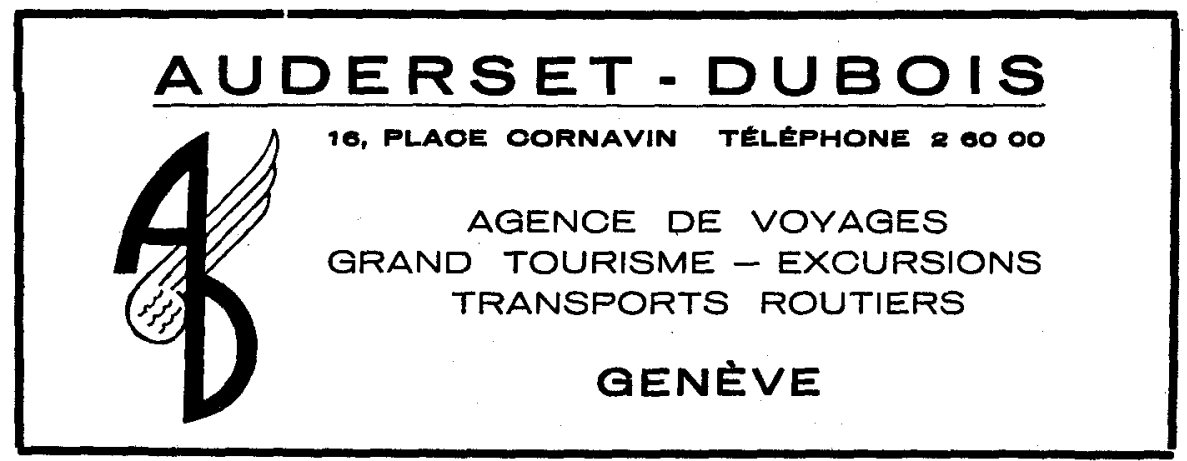




\section{BUFFETS}

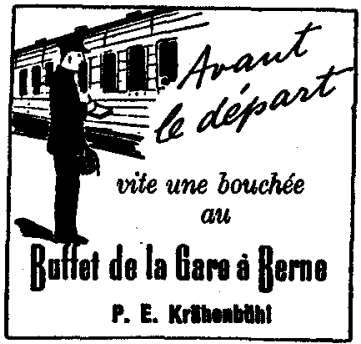

\section{De passage à ZURICH}

rendez-vous au BUFFET DE LA GARE vous y trouverez l'accueil le plus aimable une bonne table un service frompt et soigné

Vons seres regus et soignt. impeceablement

Yemes maisons:

PARK HOTEL, Vitenan (avril-nov.) SUVRETTA HOUSE, St-Morite (Engadine) (Saisons-éte ot hiver)

\section{HOTELS}

HOTEL

\section{RICHEMOND}

Près du Lac

\section{Genève}

\section{Rue Adhémar-Fabri}

Tél. 27120

H O T E L

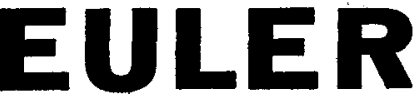

Ier RANG

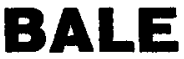

En face de la Gare CFF

Tákphone: Téjégramme :

$21723 \quad$ Eulerhotel

\section{CLINIQUES}

\section{La Clinique MONT-RIANT à Chamby sur Montreux}

Speclalement organisé pour

les cures de repos, les convalescences et le traitement.

des maladies chroniques vous offre

des chambres avec loggias, des chambres au soleil avec et sans balcons, des appartements avec salle de bain, une cuisine soignée et une situation priviléglbe au prix de Fr. 14,- - 28,- par jour. (Arrangements pour longs séjour.) Dr G O D A R D, médecin-dírecteur 


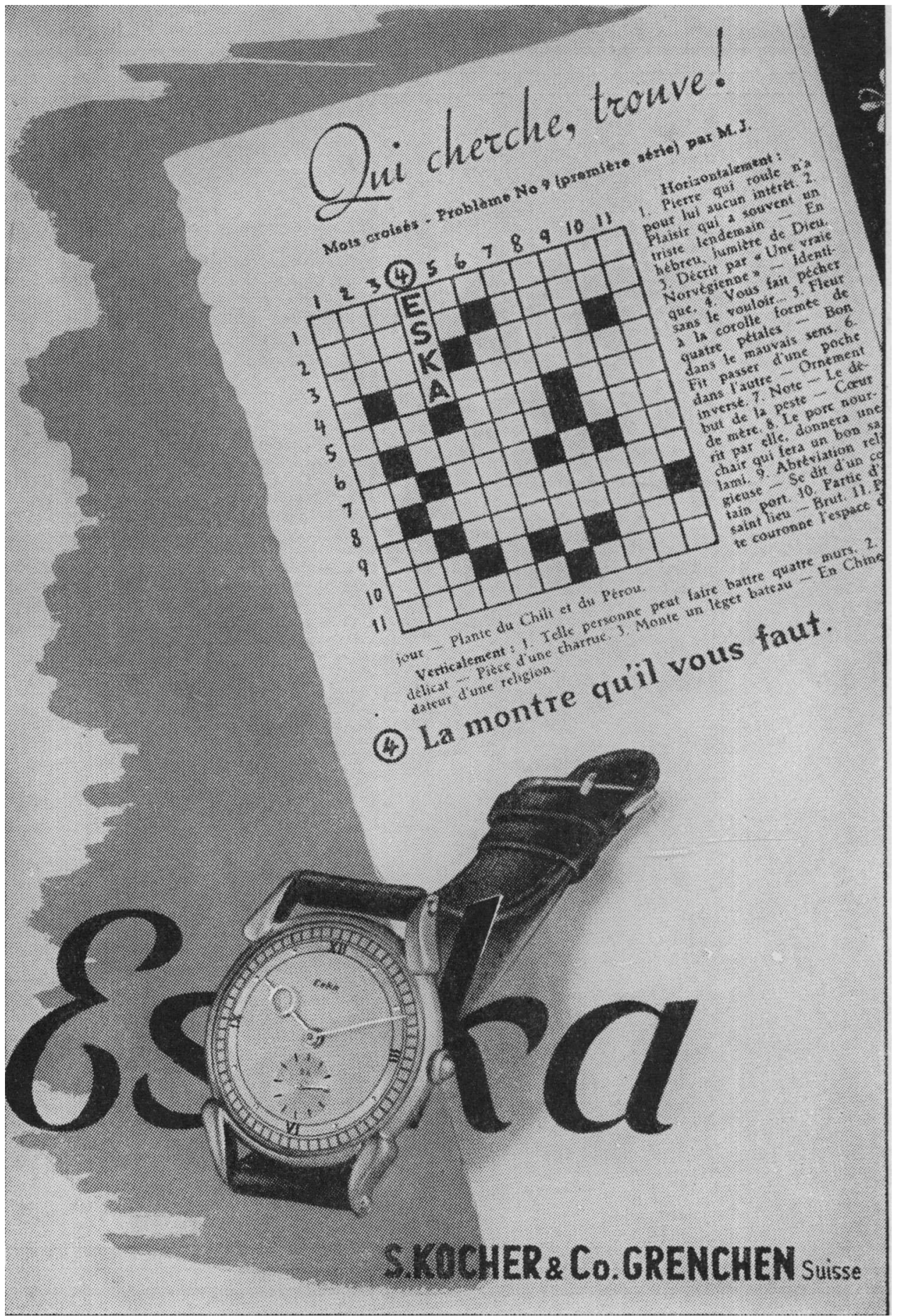



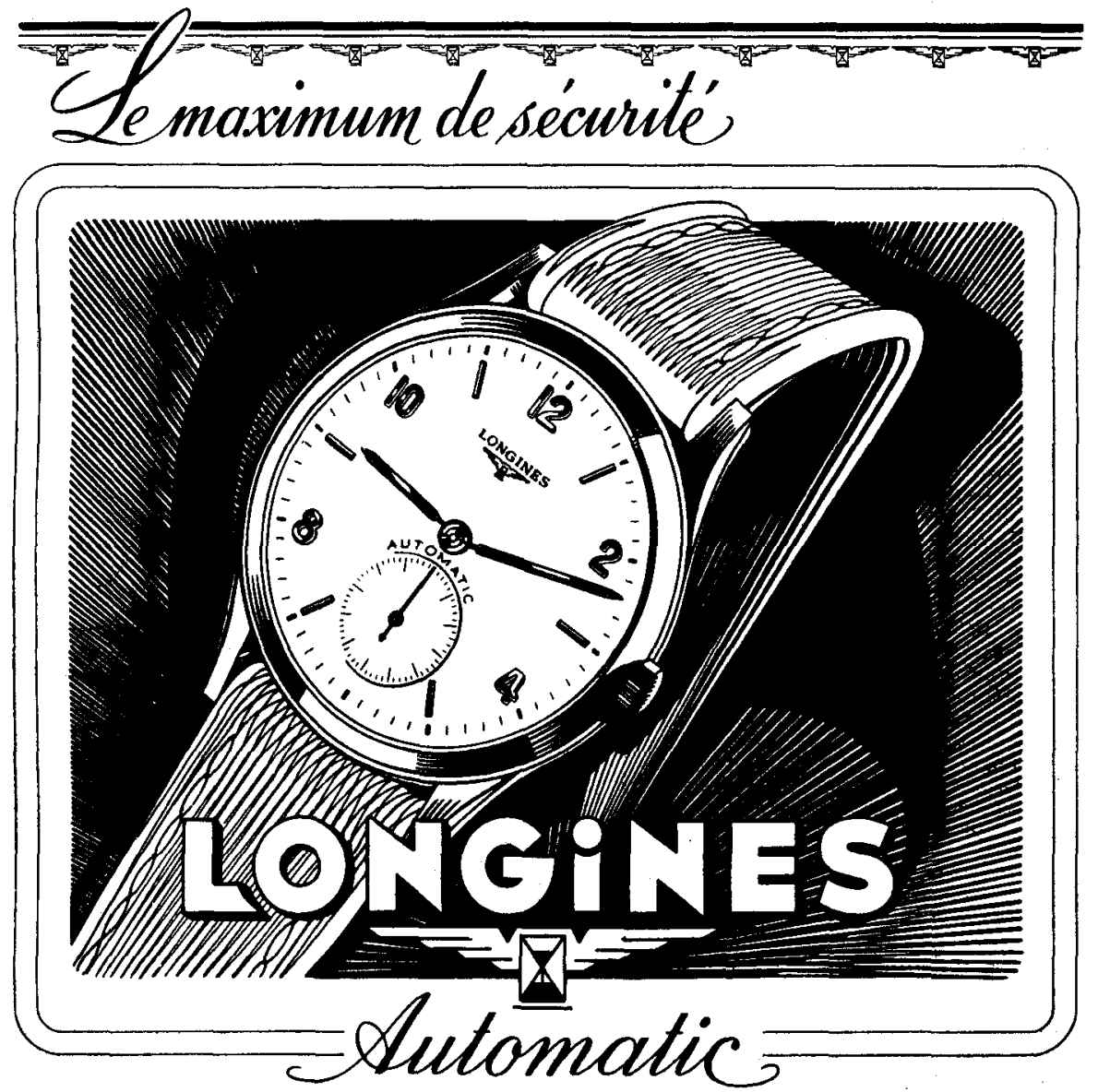

La montre automatique Longines se remonte d'elle-même, silencieusement, en moins de 3 heures et sa réserve de marche dépasse 36 heures. Equipée d'un double système de

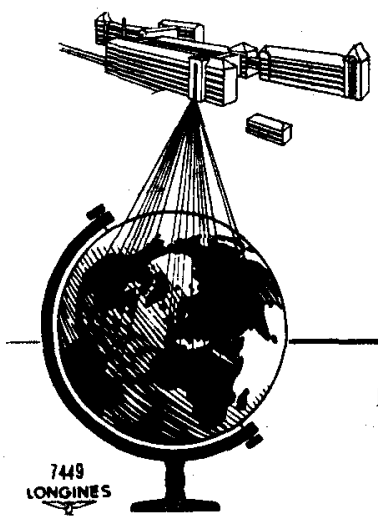
protection contre les chocs, elle est, de plus, dotée d'une stabilité de réglage remarquable.

Antimagnétique, protégée contre la poussière et l'humidité, "Longines Automatic" illustre les qualités «Longines» jusque dans ses moindres détails.

LA COMPAGNIE DES MONTRES LONGINES ET SES AGENTS DANS LE MONDE ENTIER VOUS ASSURENT LA GARANTIE DU SERVICE MONDIAL LONGINES.

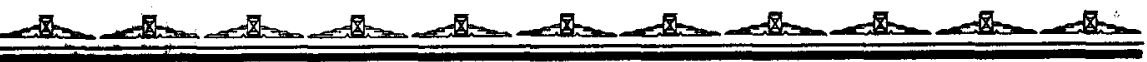




\section{ADRESSES DES COMITÉS CENTRAUX DES SOCIETÉS NATIONALES DE LA CROIX-ROUGE}

ALBANIE - Croix-Rouge albanaise, Tirana.

ALLEMAGNE - Les groupements régionaux de la CroixRouge ont formé des Comités de coordination dans la zone américaine (Junghofstrasse 26, Franctort/M.), dans la zone britannique (Harvestehuderweg 26, Hambou'g 13) et dans la zone française (Maximilianstr. 1, Baden-Baden). Il existe aussi des groupements régionaux dans la Sarre et dans la zone du Grand-Berlin.

ARGENTINE - Croix-Rouge argentine, Hipolito Irigoyen 2068, Bucnos-Aires.

AUSTRALIE - Croix-Rouge australienne, 122-128, Flinders Street, Melboume.

AUTRICHE-Croix-Rouge autrichienne,Milchgasse, 1 ienue I BELGIQUE - Croix-Rouge de Belgique, 98, Chaussée de Vleurgat, Bruxelles.

BIRMANIE - Croix-Rouge de Birmanie, Office of the director of public Health, Pbayre Street, Rangoon.

BOLIVIE - Croix-Rouge bolivienne, Casilla num. 741, La Paz.

BRESIL - Croix-Rouge brésilienne, Praça da Cruz Vermelha, 10-12, Rio de Janciro.

BULGARIE - Croix-Rouge bulgare, 21, boul. Totleben Sofia

CANADA - Croix-Rouge canadienne, 95, Wellesley Street. Toronto 5 .

CHILI - Croix-Rouge chilienne, Calle Catedral, 1572, Correo 15, Casilla, 246. V., Santiago de Chile.

CHINE - Croix-Rouge chinoise, 275, Chungsan Lu Road, Nankin.

COLOMBIE - Croix-Rouge colombienne, Calle 8a, num. 7-73, Apartado, nacional 1110, Bogota.

COSTA-RICA - Croix-Rouge costaricienne, Calle 5a Sur, San Jose.

CUBA - Croix-Rouge cubaine, Zulueta, 461, La Havane.

DANEMARK - Croix-Rouge danoise, Amaliegade, 18, Copenhague $K$.

REPUBLIQUE DOMINICAINE - Croix-Rouge dominicaine Calle Galvan, 24, Apartado 1288, Cuilad Trujillo.

ÉGYPTE - Société nationale Fouad 1 or du Croissant-Rouge égyptien, rue Malika Nazli, 34 , Le Caire.

EQUATEUR - Croix-Rouge équatorienne. A venida Colombia y Elizalde, 118, Quito.

ESPAGNE - Croix-Rouge espagnole, Eduardo Dato,18, Madrid.

ETATS-UNIS - Croix-Rouge américaine, National Headquarters, 17 th and D. Streets, N.W., Washington 13, D.C.

ETHIOPIE - Croix-Rouge éthiopienne, Addis-AbCba.

FINLANDE - Croix-Rouge de Finlande, Tebtaankatu I A, Helsinki.

FRANCE - Croix-Rouge française, 17, rue Quentin-Bauchart, Paris $\left(8^{\mathrm{me}}\right)$.

GRANDE-BRETAGNE - Croix-Rouge britannique, 14, Grosvenor Crescent, Londres S. W.1.

GRECE - Croix-Rouge hellénique, rue Mackenzie-King, 1 et rue Solonos, 39, Athènes.

GUATEMALA - Croix-Rouge du Guatémala, 4a, CalleOriente, 64, Guatémala.

HÄITI - Croix-Rouge haïtienne, rue Férou, Port-au-Prince

HONDURAS - Croix-Rouge du Honduras, Tegucigalpa.

HONGRIE - Croix-Rouge hongroise, Baross utca, 15, Budapest VIII.

INDE - Croix-Rouge de l'Inde, no 20, Talkatora Road, New Delhi 3.
IRAK - Croissant-Rouge de l'Irak, Bagdad.

IRAN - Société du Lion et Soleil Rouges de l'Iran, Avenue Argue, Théran.

IRLANDE- Croix-Rouge irlandaise, 25 Westland Row, Dublin.

ISLANDE - Croix-Rouge islandaise, Thorvaldsensstraeti, 6 Reykjavik.

ITALIE - Croix-Rouge italienne, 12, via Toscana, Rome.

JAPON - Croix-Rouge japonaise, 5 Shiba Park, Tokio.

JORDANIE - Croissant-Rouge jordanien, Amman.

LIBAN - Croix-Rouge libanaise, Beyrouth.

LIECHTENSTEIN - Croix-Rouge du Liechtenstein, Vadus.

LUXEMBOURG - Croix-Rouge luxembourgeoise, 5 , rue de Rollingergrund, Luxembourg.

MEXIQUE - Croix-Rouge mexicaine, Esq. Durango y Monterrey Col. Roma, $M$ exico DF.

MONACO - Croix-Rouge monégasque, Monaco.

NICARAGUA-Croix-Rouge du Nicaragua, Managua D.N.C.A.

NORVtGE - Croix-Rouge de Norvege, Bankplassen, 1. Oslo.

NOUVELLE-ZÉLANDE - Croix-Rouge néo-zélandaise, 61, Dixon Street, Wellington C.1.

PAKISTAN - Croix-Rouge du Pakistan, Katrak Building, Mansfield Road, Karachi $I I I$.

PANAMA - Croix-Rouge de Panama, Panama.

PARAGUAY - Croix-Rouge paraguayenne, Avenida Espana, 505, Asuncion.

PAYS-BAS - Croix-Rouge néerlandaise, 27, Princessegracht, La Haye.

PEROU - Croix-Rouge péruvienne, Avenida Nicolas de Piérola, 224, Lima.

PHILIPPINES - Croix-Rouge philippine, 106, San Raphael Street, Manille.

POLOGNE - Croix-Rouge polonaise, Mokotowska, I4, Varsovie.

PORTUGAL - Croix-Rouge portugaise, Secrétariat général Praça do Cornércio, 1, Lisbonne.

ROUMANIE - Croix-Rouge roumaine, Strada Biserica Amzei, 29, Bucarest.

SALVADOR - Croix-Rouge du Salvador, 3a, Calle Poniente, 21, San Salvador.

SIAM - Croix-Rouge siamoise, King Cbulalongkorn Memorial Hospital, Bangkok.

SUEDE - Croix-Rouge suédoise, Artillerigatan, 6, Stockholm 14.

SUISSE - Croix-Rouge suisse, Taubenstrasse, 8, Berne.

SYRIE - Croissant-Rouge syrien, Damas.

TCHECOSLOVAQUIE - Croix-Rouge tchécoslovaque Thunovska, 18, Prague III.

TURQUIE - Société du Croissant-Rouge turc, Yenisehir, Ankara.

UNION SUD-AFRICAINE - Croix-Rouge sud-africaine His Majesty's Building, Eloff Street. P.O. Box 8726 . Johannesótirg.

U.R.S.S. - Alliance des Sociétés de la Croix-Rouge et du Croissant-Rouge de l'U.R.S.S., rue Neglinnaya, 25, M oscou.

URUGUAY - Croix-Rouge uruguayenne, Avenida, 8 de Octubre, 2990, Montevideo.

VENEZUElA - Croix-Rouge vénézuélienne, Avenjda Este 303-:- Caracas.

YOUGOSLAVIE - Croix-Rouge yougoslave, Simina ulica broj. 10. Belgrade 